\title{
Evaluación de la información sanitaria disponible en internet sobre la vacuna antigripal
}

\author{
Evaluation of health information about the influenza vaccine \\ on internet
}

\author{
I. Hernández-García ${ }^{1}$, R. González Celador ${ }^{2}$
}

\section{RESUMEN}

Fundamento. La calidad de la información sanitaria en internet preocupa a gobiernos y usuarios. Nuestro objetivo fue determinar en qué medida la información sobre la vacuna antigripal se adhiere al gold estándar representado por lo establecido por el Ministerio de Sanidad español.

Material y métodos. Entre junio-julio 2014 se evaluó la adhesión al gold estándar de la información sobre indicaciones, efectos adversos y contraindicaciones de la vacuna. Dicha información se obtuvo a través de Google, utilizando términos proporcionados por estudiantes de medicina. Se realizó un análisis univariante, donde variable resultado fue obtener información correctamente adherida, y variables explicativas fueron el tipo de origen de la información y su país.

Resultados. Con los términos de 104 alumnos se obtuvieron 134 enlaces web diferentes. La adhesión alcanzó el $65,7 \%(88 / 134)$ en cuanto a la indicación en trabajadores sanitarios (TS). El 19,4\% aportaron información incorrecta sobre indicación en embarazadas. La información de organismos oficiales de salud pública se adhirió significativamente mejor (indicación en TS (OR: 2,6), embarazadas (OR: 5,4) e inmunodeprimidos (OR: 2,2). La información de enlaces web españoles se adhirió peor (indicación en embarazo (OR: 0,3$)$ y contraindicación si alergia al huevo (OR: 0,5).

Conclusiones. El nivel de adhesión detectado fue mejorable. Se debe urgir y promocionar en los usuarios la utilización de las páginas web de organismos oficiales de salud pública cuando busquen información sobre la vacuna antigripal en internet.

Palabras clave. Vacuna antigripal. Internet. Información. Evaluación.

\begin{abstract}
Background. The quality of health information on internet is a question of concern to governments and users. Our aim was to determine the extent to which information about the influenza vaccine adheres to the gold standard set by the Spanish Health Ministry.
\end{abstract}

Methods. Between June and July 2014 information on indications, adverse effects and counter-indications of the vaccine was evaluated to see if it adhered to this gold standard. This information was obtained through Google, using terms provided by medical students. A univariate analysis was performed, where the variable result was getting information that adhered correctly to the standard, and explanatory variables were the type of origin of the information and its country.

Results. Using the terms provided by 104 students, we obtained 134 different web links. Adhesion reached $65.7 \%(88 / 134)$ with respect to indication in health workers (HW). Nineteen point four percent provided incorrect information on indication in pregnant women. There was significantly better adherence in the information from official public health bodies (indication in HW (OR: 2.6), pregnant women (OR: 5.4) and immunodepressed patients (OR: 2.2). Adherence of information on Spanish web links was worse (indication in pregnancy (OR: 0.3 ) and counter-indication if allergic to eggs (OR: 0.5).

Conclusions. Adhesion was improvable. It's necessary to promote that internet users use official public health bodies websites when they search information regarding influenza vaccine on internet.

Key words. Influenza vaccines. Internet. Information. Evaluation.
1. Servicio de Medicina Preventiva y Salud Pública. Hospital Universitario Infanta Leonor Hospital Virgen de la Torre de Madrid.

2. Departamento de Medicina Preventiva, Salud Pública y Microbiología Médica. Universidad de Salamanca.

Recepción: 5 de septiembre de 2014

Aceptación provisional: 3 de noviembre de 2014

Aceptación definitiva: 18 de noviembre de 2014

\section{Correspondencia:}

Ignacio Hernández-García

Servicio de Medicina Preventiva y Salud Pública Hospital Universitario Infanta Leonor - Hospital Virgen de la Torre de Madrid Avenida Gran Vía del Este, 80 28031 Madrid. España

E-mail: ignaciohernandez79@yahoo.es

Los autores declaran que no existen conflictos de intereses. 


\section{INTRODUCCIÓN}

Internet constituye una importante fuente de información sanitaria en países occidentales; de hecho, en Estados Unidos, se estima que el $59 \%$ de las personas mayores de 18 años buscan información de este tipo a través de la red, lo cual representa la tercera actividad on line más popular ${ }^{1}$. Asimismo, en España, Tuells y col han descrito cómo la población general utiliza frecuentemente la red como fuente de información sobre vacunas ${ }^{2}$.

Internet se ha considerado como la segunda fuente de información más influyente en la toma de decisiones de los pacientes, por detrás de las recomendaciones aportadas por trabajadores sanitarios (TS) ${ }^{3}$. Una de sus principales características es que cualquier persona puede publicar información en ella; este hecho justifica la gran heterogeneidad existente en la calidad de la información sanitaria disponible en la red $^{4}$, y que desde hace años ya ha sido evidenciada en diversas revisiones, como la realizada por Cline y col, en la que describieron cómo hasta el 90,2\% de las páginas web con información sobre el tratamiento de determinadas enfermedades no se adherían a las correspondientes recomendaciones terapéuticas de las principales guías clínicas $^{5}$. Por este motivo, esta situación ha sido, y es, objeto de preocupación tanto por parte de sociedades científicas como de gobiernos y usuarios ${ }^{4}$.

En particular, la evaluación de la concordancia de la información disponible en internet sobre vacunas, respecto a la correspondiente información oficial, se ha efectuado para la vacuna antigripal ${ }^{6}$, la del papiloma humano ${ }^{7}$ o la triple vírica ${ }^{8}$, en cuanto $\mathrm{a}$, específicamente, indicación en $\mathrm{TS}^{6}$, efectividad ${ }^{7}$ y seguridad ${ }^{7,8}$. Dado que el análisis pormenorizado del contenido de la información sobre la vacuna antigripal no ha sido llevado a cabo en nuestro medio, se decidió realizar esta investigación con el objetivo de determinar en qué medida la información que se encuentra en internet sobre sus indicaciones, efectos adversos y contraindicaciones, se adhiere a lo establecido por el Ministerio de Sanidad de nuestro país.

\section{MATERIAL Y MÉTODOS}

En febrero de 2012, durante la distribución de un cuestionario autocumplimentado en alumnos de medicina de la Universidad de Salamanca, con el que se evaluó su intención de vacunarse frente a gripe en su futuro ejercicio profesional ${ }^{9}$, también se solicitó que escribieran los términos de búsqueda que utilizarían en Google para consultar información sobre dicha vacuna. Entre junio y julio de 2014, desde una dirección IP española, se introdujeron en el motor de búsqueda Google los mencionados términos, y se procedió a seleccionar los 10 primeros enlaces web de cada búsqueda, categorizándolos según la tipología de su origen (a) organismos oficiales de salud pública b) sociedades científicas c) medios de comunicación digital d) universidades e) hospitales públicos f) organizaciones sanitarias colegiales g) laboratorios h) movimientos antivacunas i) enciclopedias generales j) enciclopedias médicas $\mathrm{k}$ ) artículos de revistas indexadas en bases biomédicas l) instituciones/facultativos prestadores de asistencia sanitaria privada y m) otros) y según el país del que procedieran (España o extranjero).

La información, obtenida haciendo hasta 4 clicks en los distintos subenlaces de cada enlace tal y como han hecho otros autores $^{6}$, se recogió en una plantilla de extracción de datos en lo que respecta a los siguientes seis aspectos relacionados con las vacunas antigripales utilizadas más frecuentemente en España (vacunas inactivadas trivalentes fabricadas a partir de virus cultivados en huevos embrionados de gallina) ${ }^{10}:$ 1) indicación en TS 2) indicación en embarazadas en cualquier trimestre de la gestación 3) indicación en inmunodeprimidos 4) postponer la vacunación si existe enfermedad aguda con fiebre alta (EAFA) 5) contraindicación en alérgicos al huevo y 6) imposibilidad de que produzca la gripe.

Se evaluó si la información se adhería al gold estándar representado por la aportada por el Ministerio de Sanidad de España ${ }^{10} ;$ los enlaces web obtenidos a través de más de una búsqueda fueron considerados como solo uno. Se calcularon frecuencias absolutas y relativas para describir la adecuación de la información hallada (correcta, inco- 
rrecta o no consta); de esta forma, además de estudiar la frecuencia con la que los usuarios encontraban información correcta, también se analizó la frecuencia con la que se suscitaba error en los usuarios (información incorrecta), así como la dificultad que tenían los usuarios de encontrar información correcta (al hallar, en su lugar, información incorrecta/no consta). Además, se realizaron tres análisis univariantes en los que se consideraron las siguientes variables resultado: a) obtención de información correcta o incorrectamente adherida, b) obtención de información correctamente adherida o no consta, y c) obtención de información correctamente adherida o incorrectamente/no consta. Como variables explicativas se consideraron el tipo de origen de la información (categorizada en organismos oficiales de salud pública y el resto) y su país de procedencia. Los tests estadísticos usados fueron la prueba chi cuadrado o, en su caso, el test exacto de Fisher, cuantificándose la asociación con la odds ratio (OR) y sus intervalos de confianza del 95\% (IC 95\%). En caso de encontrarse asociación significativa entre las variables mencionadas, se realizó un análisis de regresión logística múltiple. El nivel de significación estadística considerado en todos los contrastes de hipótesis fue $\mathrm{p}<0,05$.

\section{RESULTADOS}

Los términos de búsqueda (y el número de ocasiones) facilitados por 104 estudiantes fueron: "vacuna gripe" (21), "gripe" (13), "vacuna de la gripe" (11), "vacuna antigripal" (10), "vacunación antigripal" (7), "influenza vaccine" (6), "vacuna contra la gripe" (6), "gripe A" (6), "vacunación para la gripe" (5), "vacunas contra gripe" (5), "campaña de vacunación antigripal" (4), "vacunas para la gripe" (3), "flu vaccine" (3), "información sobre la gripe" (2), "vacuna virus de la gripe" (2), "vacuna" (2), "vacunas" (2), "vacunación gripe" (2), "vacuna gripe efectos adversos" (2), "vacunas gripe" (2), "gripe, vacuna, información" (1), "vacuna gripe contraindicaciones" (1), "vacuna gripe contraindicación" (1), "vacuna antigripal beneficios" (1), "gripes" (1), "calendario vacunación" (1), "virus gripe" (1) y "gripe vacunación indicaciones" (1).

El número de enlaces web seleccionados fue 134 (Tabla 1); la adhesión al gold

Tabla 1. Direcciones electrónicas de algunos de los 134 enlaces web objeto de evaluación

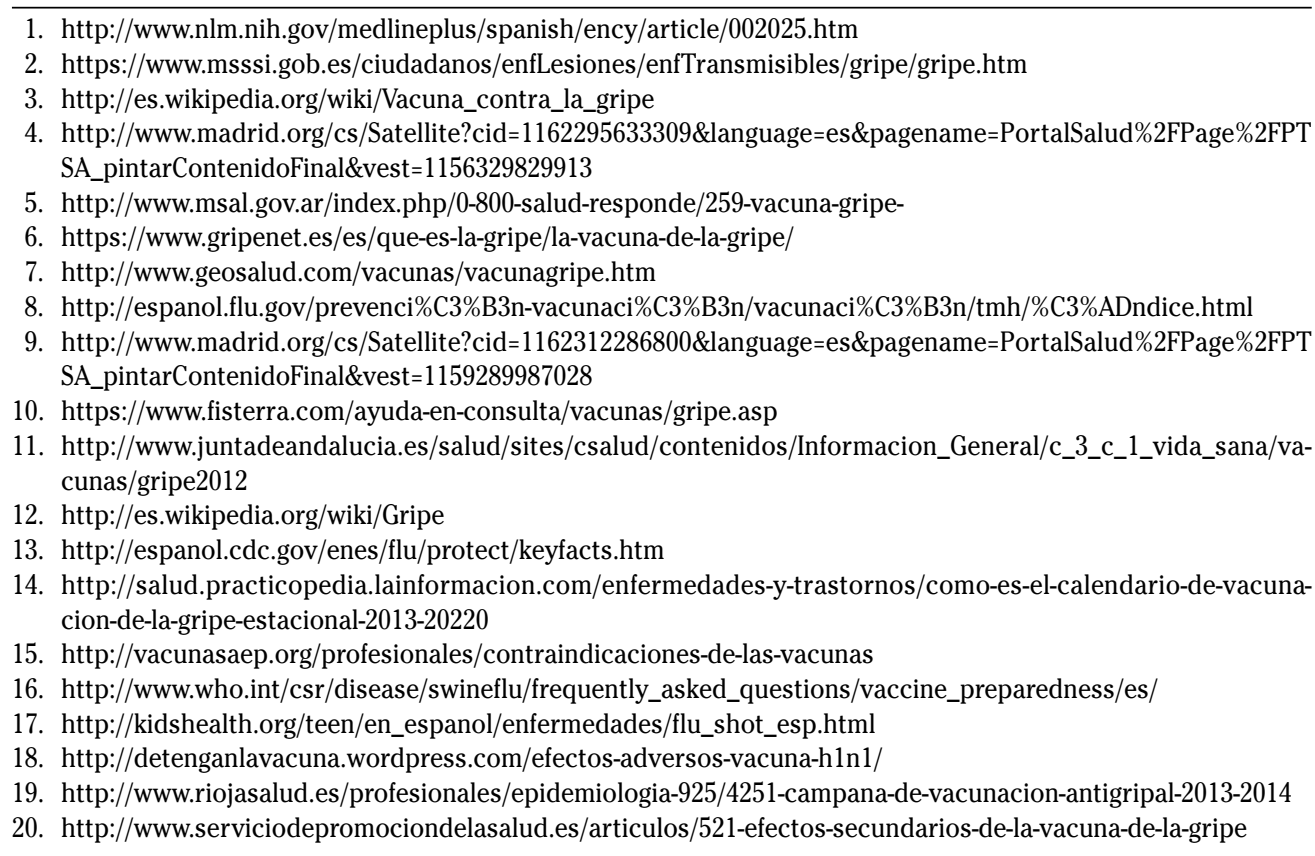


estándar de la información que proporcionaron se describe en la tabla 2. En ella se observa cómo osciló entre el $65,7 \%$ (88/134) de los enlaces en los que, de manera correcta, constaba la indicación de vacunación en TS, y el $44 \%$ en los que constaba la necesidad de postponer la administración de la vacuna si existe EAFA. Por su parte, la información que con más frecuencia fue detectada como incorrecta fue la relativa a la indicación en cualquier trimestre del embarazo (19,4\% de los enlaces aportaron información incorrectamente adherida al gold estándar). Según el tipo de origen, los enlaces web de enciclopedias médicas aportaron información correcta en todos los aspectos considerados, mientras que los de los movimientos antivacunas fueron los que suministraron mayor cantidad de información incorrecta (principalmente al indicar la no vacunación en TS y en personas inmunodeprimidas, así como al reflejar que la vacuna puede producir la gripe).

En los tres análisis univariantes, la información facilitada por organismos oficiales de salud pública se adhirió al gold estándar de una manera significativamente mejor en lo que respecta a la indicación de vacunación en cualquier trimestre de la gestación (Tabla 3). En particular, en el análisis univariante en el que se consideró variable resultado la obtención de información correcta o incorrectamente adherida a la aportada por el Ministerio de Sanidad español, también se evidenció cómo los organismos oficiales de salud pública proporcionaron información significativamente mejor en lo referente a la indicación en TS e inmunodeprimidos; por su parte, la información facilitada por enlaces web españoles se adhirió significativamente peor en lo relativo a la indicación en embarazadas y en personas inmunodeprimidas (Tabla 3).

En el análisis multivariante, las variables tipo de origen (organismos de salud pública) y país de procedencia (España) de la información sobre la indicación de la vacuna antigripal en cualquier trimestre del embarazo mantuvieron su significativamente mejor y peor adhesión al gold estándar cuando se consideró como variable resultado el obtener información correcta o incorrecta/no consta (OR: 5,2 [IC 95\%: 2,4-11,6] y OR: 0,3 [IC 95\%: 0,1-0,6], respectivamente), así como cuando se consideró como variable resultado el obtener información correcta o incorrecta (OR: 22,0 [IC 95\%: 4,5-107,6] y OR: 0,1 [IC 95\%: 0,02-0,33], respectivamente). Por su parte, solo la variable tipo de origen (organismos de salud pública) mantuvo su asociación significativa con proporcionar con más frecuencia información correcta que incorrecta en relación a la indicación en inmunodeprimidos (OR: 10,2 [IC 95\%: 1,2-87,1]).

\section{DISCUSIÓN}

Este trabajo representa, hasta nuestro conocimiento, el primer estudio en el que se ha evaluado el nivel con el que se adhiere la información disponible en internet, sobre la vacuna antigripal, a lo indicado por el Ministerio de Sanidad español. En él se ha evidenciado un nivel de adhesión mejorable, y una dificultad para obtener información correctamente adherida, dado que la misma solo se obtuvo en el 44\%-65,7\% de los enlaces, según el tipo de información buscada. Dicho porcentaje de adhesión fue inferior al obtenido por otros autores, tales como Betsch y col en Alemania ${ }^{6}$, quien describió cómo el 88,5\%, de las páginas web que evaluó aportaban información correcta sobre la indicación de vacunación en TS de acuerdo a las recomendaciones oficiales del German Standing Committee on Vaccination, STIKO. Además, que menos de dos terceras partes de los enlaces analizados hayan aportado información correcta sobre la indicación de vacunación en TS es relevante, dado las bajas coberturas de vacunación que suelen registrarse en nuestro medio en este grupo específico de la población ${ }^{11}$ y dado que conocer dicha indicación se ha observado que se asocia positivamente con la intención de los estudiantes sanitarios de vacunarse frente a la gripe ${ }^{12}$.

Por su parte, otro autor que ha obtenido mejores resultados fue Covolo y $\mathrm{col}^{13}$, quienes describieron cómo en el $80,3 \%$ (61/76) y 53,9\% (41/76) de las páginas web que evaluó constaban, respectivamente, información sobre las indicaciones y con- 


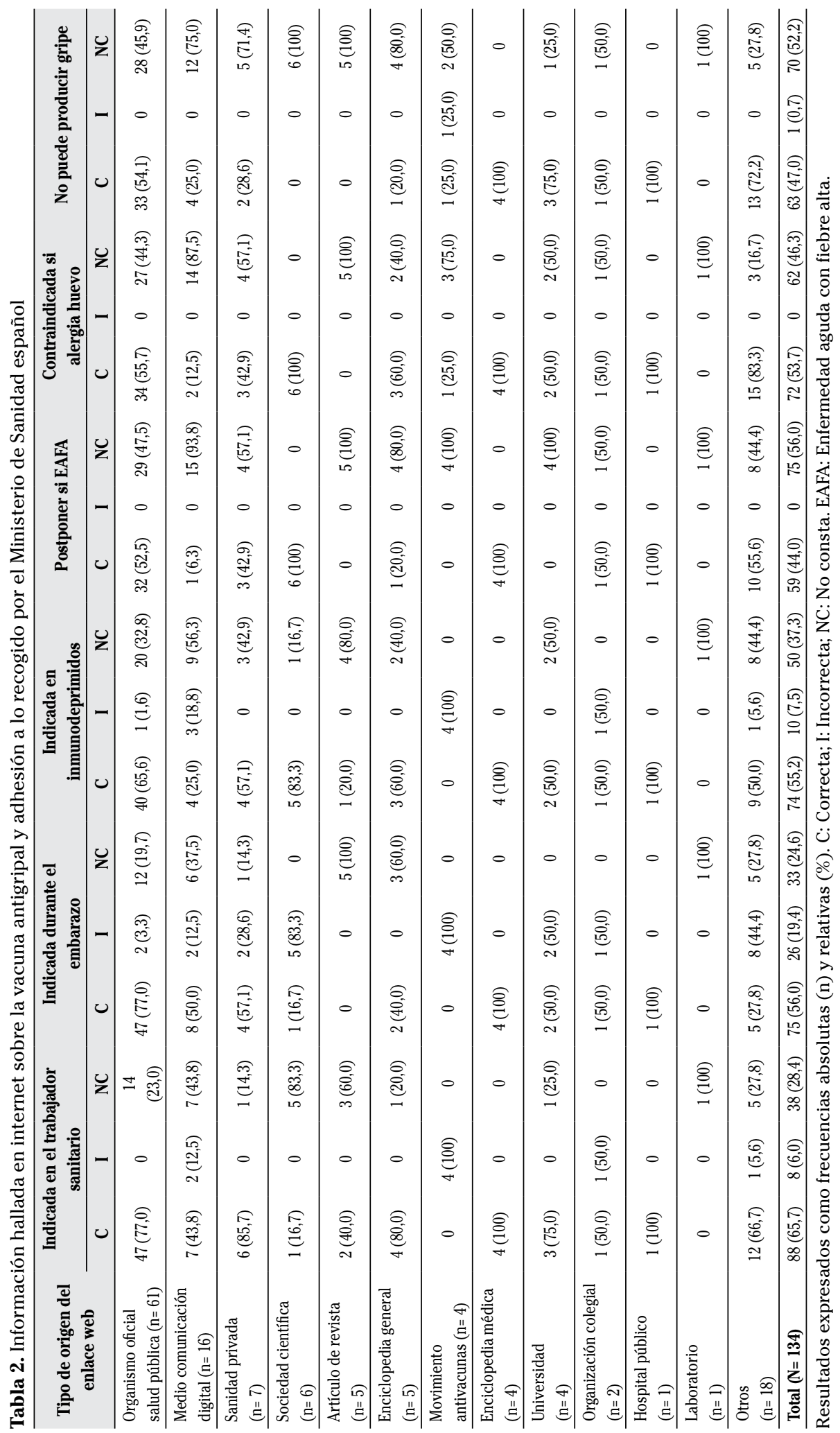




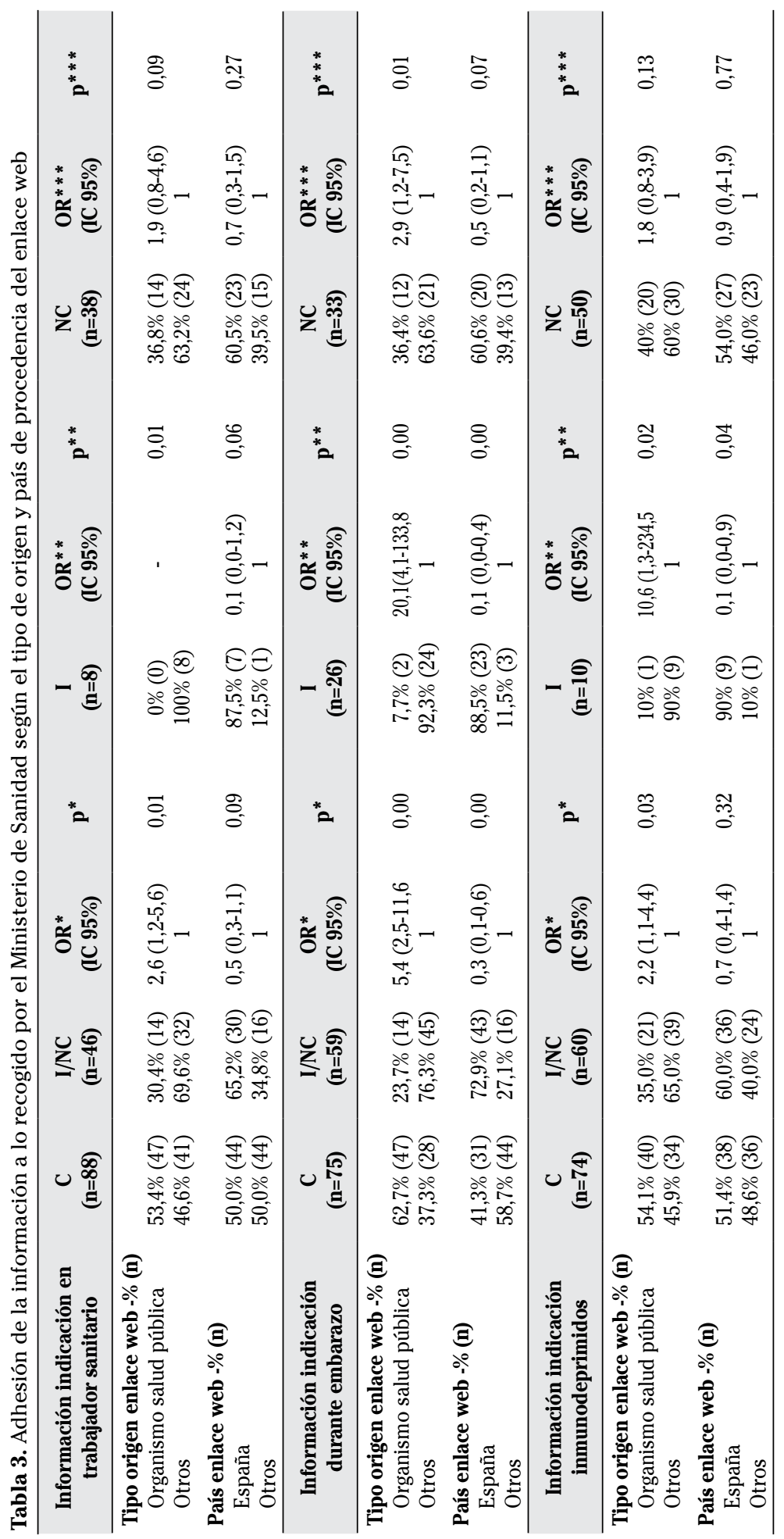




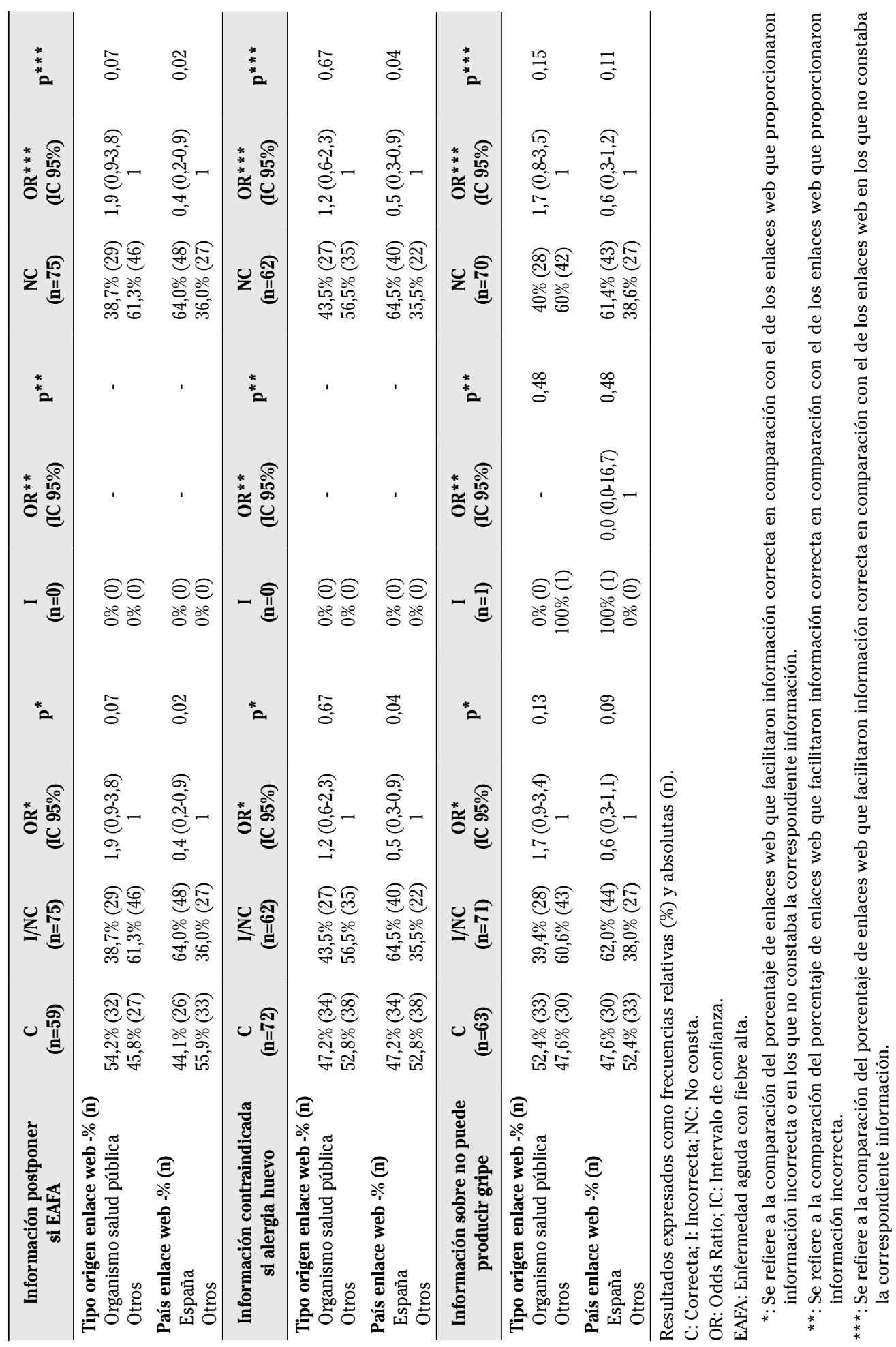


traindicaciones de la vacuna adheridas correctamente a las recomendaciones de la Organización Mundial de la Salud (las cuales incluyen la indicación en TS, embarazadas e inmunodeprimidos, así como la contraindicación en alérgicos al huevo). Entre los factores que podrían justificar las mencionadas diferencias se encontraría el idioma utilizado para realizar las búsquedas en Internet; así, Betsch y Wicker ${ }^{6}$ utilizaron los términos de búsqueda proporcionados por 310 estudiantes de medicina de la Universidad de Frankfurt, mientras que Covolo y col $^{13}$ excluyeron de su estudio las páginas web no disponibles en inglés; todo ello conlleva que la información evaluada en dichos estudios muy previsiblemente no haya tenido su origen en España, lo cual, tal y como se ha evidenciado en nuestra investigación, se asocia con proporcionar con menos frecuencia información correcta, que información incorrecta o incorrecta/no consta o no consta.

Por otro lado, y en particular, que únicamente el $47 \%$ de los enlaces evaluados hayan aportado información correcta sobre que la vacuna no puede producir la gripe es especialmente preocupante en nuestro medio, dado que dicho motivo ha sido registrado como una de las principales razones alegadas por los TS para no vacunarse ${ }^{14}$.

El hecho de que los organismos oficiales de salud pública, de manera significativa, hayan aportado con más frecuencia información correcta, que incorrecta o incorrecta/no consta, sobre las indicaciones objeto de estudio, confirma lo señalado por otros autores en cuanto a la fiabilidad de la información aportada por tales instituciones $^{6,13}$. Por contra, los enlaces de los movimientos antivacunas destacaron por la cantidad de información incorrecta que suministraron, basándose para ello en los típicos argumentos que estos grupos suelen utilizar en cuanto a falta de efectividad de las vacunas y la posibilidad de que causen la enfermedad ${ }^{15}$.

La frecuencia con la que se obtuvo información netamente incorrecta, principalmente en lo relacionado con la indicación de la vacunación en cualquier trimestre del embarazo, supuso un hallazgo particu- larmente importante, dado que en nuestro medio las coberturas de vacunación en embarazadas son muy bajas $(5,7 \%)^{16}$, y se ha descrito cómo únicamente el 57,1\% de los médicos de familia recomiendan la vacuna antigripal durante el primer trimestre del embarazo ${ }^{11}$. Además, cabe destacar que varios enlaces de sociedades científicas, como el de la Asociación Española de Pediatría ${ }^{17}$, no se adhirieron a la indicación de vacunar a la mujer embarazada en cualquier trimestre de la gestación, al recoger la siguiente matización: "En general las vacunas inactivadas se podrían aplicar durante el embarazo, aunque en algunas de ellas no existen estudios que ratifiquen su seguridad. Siempre hay que valorar si el beneficio es mayor que el riesgo, siendo de aplicación relativamente frecuente durante el embarazo las vacunas de toxoides, especialmente la antitetánica, la vacuna antigripal (a partir del segundo trimestre de embarazo, aunque si el riesgo grande se puede aplicar también en el primero) y la vacuna frente a la hepatitis $\mathrm{B}^{\prime 17}$. Por todo ello, consideramos que sería interesante realizar una investigación, a fin de establecer en qué medida la información disponible en internet influye en la decisión de recibir la vacuna antigripal en este grupo específico de la población de nuestro país.

Entre las limitaciones de nuestro estudio se encuentra la intrínseca a internet: la información cambia continuamente, mientras que, al igual que otros autores ${ }^{6-8,13}$, en este trabajo se analizó la que se encontraba disponible en un momento particular. Asimismo, la información se evaluó exclusivamente respecto a un tipo de vacuna antigripal (vacunas inactivadas trivalentes fabricadas a partir de virus cultivados en huevos embrionados de gallina) por ser las que se utilizaban con más frecuencia en España en general, y en Castilla y León en particular, en el momento de realización del estudio. Por este motivo, no hemos analizado el nivel de adecuación de información específica para otro tipo de vacunas antigripales, tales como las obtenidas en cultivos celulares, que en la presente campaña de vacunación 2014-2015 (implementada cuatro meses después de que rea- 
lizáramos la búsqueda en internet), se han introducido ampliamente, y que no están contraindicadas en alérgicos al huevo.

Por otro lado, la evaluación de la información se hizo sólo de los primeros 10 enlaces obtenidos, si bien, se ha observado cómo los usuarios de la red no suelen utilizar más que la primera página de resulta$\operatorname{dos}^{18}$. Asimismo, la búsqueda se realizó únicamente con el motor de búsqueda Google, dado que con los restantes buscadores se efectúan menos del 15\% del total de búsquedas $^{19}$. Finalmente, los términos de búsqueda utilizados fueron los que aportaron un grupo de usuarios de internet (estudiantes de medicina), lo cual ha supuesto que nuestros resultados presenten una buena validez, sobre todo en comparación con la de otros trabajos en los que para la búsqueda se emplearon los términos que los autores creían que eran utilizados por los usuarios de la red ${ }^{13}$.

En cualquier caso, y de acuerdo a los resultados obtenidos, los profesionales sanitarios debemos urgir y promocionar, en los usuarios, la utilización de las páginas web de organismos oficiales de salud pública cuando busquen en internet información sobre la vacuna antigripal. Con ello no solo obtendrán con más frecuencia información de alta calidad, sino que se conseguirá que tales páginas mejoren su accesibilidad y posicionamiento, dado que los buscadores justifican el posicionamiento de los enlaces obtenidos en una búsqueda en base a la frecuencia de accesos a los mismos. Por su parte, y en particular, en el caso de los estudiantes sanitarios, las universidades tienen la responsabilidad de formar el criterio de los futuros profesionales para que sepan elegir fuentes fiables para informarse.

\section{BIBLIOGRAFÍA}

1. Fox S. Health information is a popular pursuit online. Pew Research Internet Project. [Consultado 10-08-2014]: Disponible en: http:// www.pewinternet.org/2011/02/01/health-information-is-a-popular-pursuit-online/.

2. Tuells J, Caballero P, Nolasco A, Montagud E. Factores asociados a la predisposición a vacunarse contra la gripe pandémica A/H1N1 en población adulta del Departamento de Salud de Elche (España). Influencia de las fuentes de información. An Sist Sanit Navar 2012; 35: 251-260.

3. Couper MP, Singer E, Levin CA, Fowler FJ Jr, FAGERLIN A, ZiKMUND-Fisher BJ. Use of the Internet and ratings of information sources for medical decisions: results from the DECISIONS survey. Med Decis Making 2010; 30 (5 Suppl): 106S-114S.

4. MAYer MA, Leis A, SANZ F. Información sobre salud en internet y sellos de confianza como indicadores de calidad: el caso de las vacunas. Aten Primaria 2009; 41: 534-542.

5. Cline RJ, Haynes KM. Consumer health information seeking on the Internet: the state of the art. Health Educ Res 2001; 16: 671-692.

6. Betsch C, Wicker S. E-health use, vaccination knowledge and perception of own risk: drivers of vaccination uptake in medical students. Vaccine 2012; 30: 1143-1148.

7. Madden K1, Nan X, Briones R, WaKs L. Sorting through search results: a content analysis of HPV vaccine information online. Vaccine 2012; 30: 3741-3746.

8. Scullard P, Peacock C, Davies P. Googling children's health: reliability of medical advice on the internet. Arch Dis Child 2010; 95: 580-582.

9. Hernández-García I. González-Celador R, GiméNEZ-Júlvez MT. Intención de los estudiantes de medicina de vacunarse contra la gripe en su futuro ejercicio profesional. Rev Esp Salud Publica 2014; 88: 407-418.

10. Ministerio de Sanidad, Servicios Sociales e Igualdad. Prevención de la Gripe. Vacunación antigripal. [Consultado 01-06-2014]: Disponible en: https://www.msssi.gob.es/ ciudadanos/enfLesiones/enfTransmisibles/ gripe/gripe.htm\#prevencion.

11. Martínez-Baz I, Díaz-González J, Guevara M, ToLedo D, Zabala A, Domínguez A et al. Actitudes, percepciones y factores asociados a la vacunación antigripal en los profesionales de atención primaria de Navarra, 2011-2012. An Sist Sanit Navar 2013; 36: 263-273.

12. Hernández-García I, Cardoso-Muñoz A, MorenoPascual C, Sáenz-González MC. Razones de los estudiantes sanitarios para tener intención de vacunarse o no de gripe cuando sean trabajadores sanitarios. Enferm Clin 2011; 21: 300-301.

13. Covolo L, Mascaretti S, Caruana A, Orizio G, Caimi L, Gelatti U. How has the flu virus infected the Web? 2010 influenza and vaccine information available on the Internet. BMC Public Health 2013; 13: 83. 
14. Galicia-García MD, González-Torga A, GarcíaGonzález C, Fuster-Pérez M, Garrigós-Gordo I, LóPEZ-FrESNEÑA N, et al. Vacunación de gripe en trabajadores sanitarios. Por qué se vacunan y por qué no se vacunan. Enferm Infecc Microbiol Clin 2006; 24: 413-417.

15. KatA A. A postmodern Pandora's box: antivaccination misinformation on the Internet. Vaccine 2010; 28: 1709-1716.

16. Bueno Campaña M, González Spinola A, Parra Cuadrado E, Quevedo Teruel S, Calvo Rey C. Vacunación antigripal en la embarazada. Prog Obstet Ginecol 2010; 53: 293-296.
17. Asociación Española de Pediatría. Contraindicaciones de las vacunas. [Consultado 2206-2014]: Disponible en: http://vacunasaep. org/profesionales/contraindicaciones-delas-vacunas\#contraind_p

18. McTavish J, Harris R, Wathen N. Searching for health: the topography of the first page. Ethics Inf Technol 2011; 13: 227-240.

19. SEO Consultants Directory. Top Search Engines in the United Kingdom. [Consultado 03-06-2014]: Disponible en: http://www.seoconsultants.com/search-engines/uk/. 